\title{
A Dynamical Systems Model of Autoignition in the Cone Calorimeter
}

\author{
M.I. NELSON \\ Department of Fuel and Energy \\ The University of Leeds \\ Leeds, LS2 9JT England, UK
}

\begin{abstract}
A non-linear dynamical-systems model for the ignition of thermally thin thermoplastics in the cone calorimeter is investigated. The model contains equations for solid phase and gas phase processes which are coupled through heat and mass transfer. We use the model to evaluate the critical heat flux required for ignition and investigate how this depends upon the degradation kinetics of the thermoplastic. The concept of a critical surface temperature defining criticality is validated.
\end{abstract}

KEYWORDS: cone calorimeter, critical heat flux, thermal pyrolysis, thermoplastics.

\section{Nomenclature}

The subscript $s$ refers to a property of the solid phase, subscript $g$ to a property of the gas-phase, subscript 0 to the solid/volatile boundary and subscript 1 to the volatile/ambient boundary.

$\begin{array}{lll}A & \text { Pre-exponential factor. } & \left(\mathrm{s}^{-1}\right) \\ E & \text { Activation energy. } & \left(\mathrm{Jmol}^{-1}\right) \\ \mathcal{F}_{\text {g.s }} \text { and } \mathcal{F}_{s . g} & \text { Integrated configuration factors. } & (-) \\ \mathcal{H} & \text { Ramping rate used in TG experiment. } & \left(\mathrm{Ks}^{-1}\right) \\ \mathcal{L} & \text { Heat-flux from the ignition source per unit area of the } & \\ & \text { solid surface. } & \left(\mathrm{Wm}^{-2}\right) \\ \overline{\mathcal{L}_{c r}} & \text { Averaged critical heat flux for ignition for a given characteristic } \\ & \text { temperature. } & \left(\mathrm{Wm}^{-2}\right) \\ \mathcal{M}_{1} & \text { Mass of solid fuel. } & (\mathrm{kg}) \\ \mathcal{M}_{2} & \text { Mass of gaseous fuel. } & (\mathrm{kg}) \\ \mathcal{M}_{3} & \text { Mass of gaseous product. } & (\mathrm{kg}) \\ \mathcal{M}_{j}^{*} & \text { Non-dimensionalised concentrations. } & \end{array}$


$\mathcal{M}_{j}^{*}=\mathcal{M}_{j} /\left(V_{s} \rho_{s}\right)(\mathrm{j}=1,2,3)$

$\mathcal{P}^{0} \quad$ Standard atmospheric pressure.

Heat of reaction.

$\left(\mathrm{Jkg}^{-1}\right)$

Ideal gas constant.

$\left(\mathrm{JK}^{-1} \mathrm{~mol}^{-1}\right)$

Mass flow of fuel into the gaseous reaction zone. $\quad\left(\mathrm{kgs}^{-1}\right)$

Sample surface area.

$\left(\mathrm{m}^{2}\right)$

Temperature.

$(\mathrm{K})$

Non-dimensionalised temperature.

$T_{i}^{*}=T_{i} / T_{a}(\mathrm{i}=\mathrm{g}, \mathrm{i}=\mathrm{s})$

Ambient temperature.

Fuel characteristic temperature.

Time.

(s)

Volume.

$\left(\mathrm{m}^{3}\right)$

Molecular weight of species $M_{2}$.

$\left(\mathrm{kgmol}^{-1}\right)$

Molecular weight of species $M_{3}$.

$\left(\mathrm{kgmol}^{-1}\right)$

Specific heat capacity.

$c_{p}$

First order reaction rate.

$\left(\mathrm{JK}^{-1} \mathrm{~kg}^{-1}\right)$

$\left(\mathrm{kgs}^{-1}\right)$

Volumetric flow rate caused by decomposition of the solid.

$\left(\mathrm{m}^{3} \mathrm{~s}^{-1}\right)$

Volumetric flow rate produced by the gaseous reaction.

$\left(\mathrm{m}^{3} \mathrm{~s}^{-1}\right)$

Non-dimensionalised time.

$t^{*}=t S_{0} \chi_{0} /\left(c_{p_{s}} \rho_{s} V_{s}\right)$

$q_{\text {reaction }}$

$t^{*}$

Volume per kilogramme of $\mathcal{M}_{2}$ (calculated using

$(一)$

the ideal gas law).

Width of the solid.

$\left(\mathrm{m}^{3} \mathrm{~kg}^{-1}\right)$

Length of the solid.

$(\mathrm{m})$

Absorptivity.

$(\mathrm{m})$

Heat transfer coefficient.

$(-)$

$\alpha$

Heat transfer coefficient between the sides of the 'gaseous slab' and the surrounding air.

$\left(\mathrm{Wm}^{-2} \mathrm{~K}^{-1}\right)$

$\chi_{2}$

$\delta_{0}$

Solid thickness.

$\left(\mathrm{Wm}^{-2} \mathrm{~K}^{-1}\right)$

Thickness of the gaseous reaction zone.

$(\mathrm{m})$

Emissivity.

$(\mathrm{m})$

Density.

$(-)$

Stefan-Boltzmann constant.

Unless otherwise specified we take the following typical parameter values:

$A_{g}=10^{12}\left(\mathrm{~s}^{-1}\right), E_{g}=150\left(\mathrm{kJmol}^{-1}\right), \mathcal{F}_{g . s}=\mathcal{F}_{s . g}=1, \mathcal{H}=1 / 60\left(\mathrm{Ks}^{-1}\right), Q_{g}=$ $30000\left(\mathrm{kJkg}^{-1}\right), Q_{s}=-1000\left(\mathrm{kJkg}^{-1}\right), S_{0}=0.0625\left(\mathrm{~m}^{2}\right), T_{c}=620(\mathrm{~K}), V_{g}=10^{-3}\left(\mathrm{~m}^{3}\right)$ $W_{2}=0.1(\mathrm{~kg}), W_{3}=0.02(\mathrm{~kg}), c_{p_{g}} \rho_{g}=1.04 \times 10^{3}\left(\mathrm{JK}^{-1} \mathrm{~m}^{-3}\right), c_{p_{s}}=1\left(\mathrm{kWm}^{-2} \mathrm{~K}^{-1}\right), x$ $=y=0.25(\mathrm{~m}), \chi_{0}=\chi_{1}=\chi_{2}=30\left(\mathrm{Wm}^{-2} \mathrm{~K}^{-1}\right), \delta_{0}=0.001(\mathrm{~m}), \delta_{1}=0.016(\mathrm{~m})$, and $\rho_{s}=2000\left(\mathrm{kgm}^{-3}\right)$.

The appropriate values for the physical constants are $\mathcal{P}^{0}=100(\mathrm{kPa}), R=8.31441$ $\left(\mathrm{JK}^{-1} \mathrm{~mol}^{-1}\right), \epsilon_{g}=\alpha_{g}=0.1, \epsilon_{s}=\alpha_{s}=1$, and $\sigma=5.67 \times 10^{-8}\left(\mathrm{Js}^{-1} \mathrm{~m}^{-2} \mathrm{~K}^{-4}\right)$. 


\section{INTRODUCTION}

One of the aims of fire engineering mathematics is to model polymer ignition, as a means of gaining an insight into the properties that govern material flammability. The main methods used to model the ignition process define ignition in terms of solid-phase properties, i.e. a critical surface temperature or a critical mass flux rate of volatiles. These methods can be used to investigate the effectiveness of additives active in the solid-phase in reducing flammability, but cannot be extended to cover additives active in the gas-phase. Accordingly it is of interest to develop models of ignition containing both solid- and gas-phase processes.

One way to do this is to utilise a dynamical systems approach in which these processes are described by coupled non-linear equations. Although such models are more complicated than those resulting from the alternative approaches, techniques such as bifurcation theory and path-following methods can be employed to explain the ignition mechanisms and to gain an insight into the relative importance of key parameters governing flammability.

Dynamical system models describing polymer ignition have been published by Rychly and Costa[1] and Nelson et al[2]. Both systems were examined by direct integration, rather than dynamical systems methods. There are differences in how the physics and gas-phase chemistry is modelled in these papers. For example, Rychly and Costa do not explicitly model radiative heat transfer and model the irradiance source by assuming that it raises the temperature of ambient gases. Nelson et al include radiation explicitly and model the irradiance source by assuming that it heats the reaction-gases and solid sample. Further work is required to determine whether the differences in modelling are significant from the perspective of flammability and retardancy.

The aim of this paper is to extend the model of Nelson et al and to illustrate how ideas from the theory of dynamical systems can be used to investigate the critical heat flux of a thermally thin thermoplastic. The validity of the concept of a critical surface temperature governing ignition is also investigated.

\subsection{Kinetic Parameters for the Solid Degradation Reaction}

As in our earlier work[3] we define solid-phase kinetic parameters by using the concept of a characteristic temperature, as measured in thermogravimetric experiments (TG). Assuming that the solid fuel undergoes a first order thermal degradation reaction, which is described by a one-step Arrhenius reaction, the characteristic temperature, $T_{c}$ measured at a given ramping rate $\mathcal{H}$, is given by[4],

$\frac{A_{s}}{\mathcal{H}}=\frac{E_{s}}{R T_{c}^{2}} \exp \left[\frac{E_{s}}{R T_{c}}\right]$.

This equation links the pre-exponential factor $\left(A_{s}\right)$ and the activation energy $\left(E_{s}\right)$, which define the kinetics for the pyrolysis of the fuel, to the characteristic temperature $\left(T_{c}\right)$, at a given ramping rate $(\mathcal{H})$. We assume that the value for the characteristic temperature is always determined at the same ramping rate.

The full reaction rate

$k=-A_{s} \exp \left[\frac{-E_{s}}{R T}\right] M_{1}$, 
can be rewritten using equation (1) as

$k=-\frac{\mathcal{H} E_{s}}{R T_{c}^{2}} \exp \left[\frac{E_{s}}{R}\left(\frac{1}{T_{c}}-\frac{1}{T}\right)\right] M_{1}$,

where the value chosen for $T_{c}$ corresponds to a given value for $\mathcal{H}$. Note that for a fixed characteristic temperature increasing the activation energy narrows the temperature range over which the fuel decomposes.

Using the form of the rate-equation in equation (3) we investigate the dependence of ignition properties upon degradation kinetics by fixing the characteristic temperature and varying the activation energy.

\subsection{Path Following Methods}

Many mathematical models can be written in the form

$\frac{\mathrm{d} \mathbf{x}}{\mathrm{dt}}=\mathbf{f}(\mathbf{x}, \lambda, \boldsymbol{\mu})$

where $\mathbf{x}$ is a $\mathrm{n}$-dimensional vector, $\lambda$ is a 1-dimensional parameter (the primary bifurcation parameter, $\boldsymbol{\mu}$ is an m-dimensional vector comprising all other parameters in the problem (the secondary bifurcation parameters) and the nonlinear function $\mathbf{f}$ consists of $\mathrm{n}$ components. In the context of radiative ignition experiments the primary bifurcation parameter is the non-dimensionalised irradiance, whilst secondary bifurcation parameters are parameters such as fuel density and the heat of gasification.

The properties of equation (4) can often be understood by considering its steady-state solutions. These satisfy the equation

$\mathbf{f}(\mathbf{x}, \lambda, \boldsymbol{\mu})=\mathbf{0}$,

where $\mathbf{0}$ is the $\mathrm{n}$-dimensional zero vector.

Solutions to equation (5) vary with the parameter $\lambda$, generally forming continuous curves in the $(\mathbf{x}, \lambda)$-space. These curves can be obtained by the use of path-following methods. The study of equations (4-5) by such methods leads to a greater understanding of the dynamics of equation (4) then would be gained by direct integration alone. Furthermore this process is often quicker than investigation by direct integration.

Path-following has attracted much interest in conjunction with the study of nonlinear phenomena, such as bifurcation. In many combustion systems ignition can be identified with an appropriate bifurcation, for example in Figure 3 ignition is identified with the limit point in the positive quadrant (Section 4.1). This limit point defines a critical value of the primary bifurcation parameter. How this critical value changes as a secondary parameter is varied shows how sensitive the critical heat flux is to other parameters, for example its dependence upon the degradation kinetics of the fuel is shown in Figure 4.

Seydel[5-6] has provided a good introduction to the ideas of path-following methods.

\section{DESCRIPTION OF THE MODEL}

The model presented in this paper is an extension of an existing model[2]. In Sections 2.1-2.2 we provide a brief description of the physics and chemistry included in the model; a more detailed description is provided in our earlier paper. 


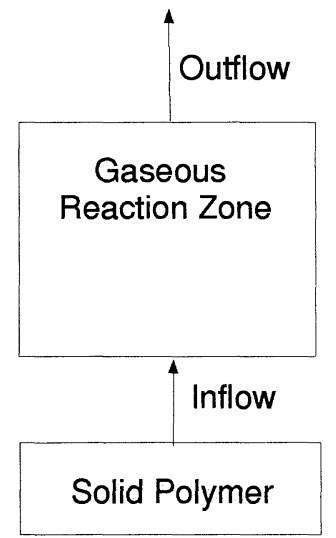

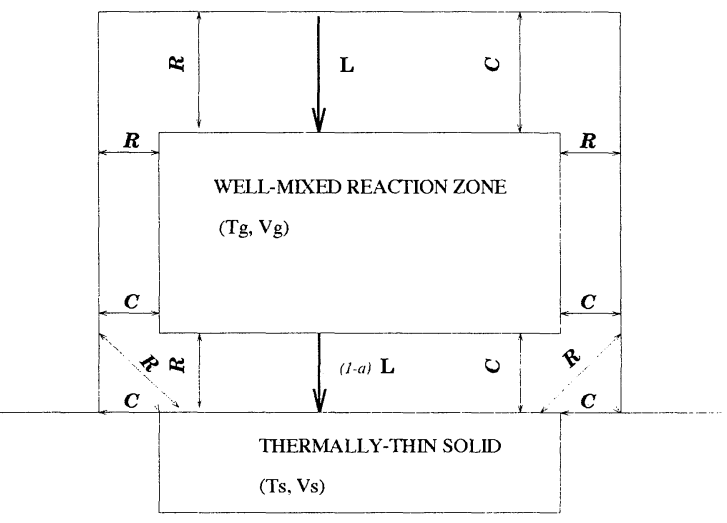

C: Conductive Heat Exchange.

a: Gas-phase absorbtivity.

Ti: Temperature.
R: Radiative Heat Exchange.

Vi: Volume.
L: External heat-flux.

Figure 1: Schematic diagrams of the model geometry illustrating inflow/outflow and of the heat-transfer processes modelled.

\subsection{Description of Physics and Chemistry of the Model}

For mathematical convenience the sample is assumed to be a thermally thin thermoplastic, the bottom face and sides of which are perfectly insulated. The sample, the gaseous reaction zone, and gases at ambient temperatures undergo convective and radiative heat exchange as indicated in Figure 1. The degree of radiative coupling between these zones is controlled by the integrated configuration factors $\left(\mathcal{F}_{s . g}\right.$ and $\left.\mathcal{F}_{g . s}\right)$. The gas-phase is assumed to be a uniform black body with constant emissivity (absorptivity).

The chemistry is described by two first-order thermal degradation reactions. In the solid-phase the fuel $\left(\mathcal{M}_{1}\right)$ decomposes endothermically producing the gaseous fuel $\left(\mathcal{M}_{2}\right)$ which flows into the gaseous reaction zone where it decomposes exothermically, releasing gaseous products molecules $\left(\mathcal{M}_{3}\right)$. The assumption that the the gaseous reaction is first order effectively assumes that oxygen is in excess in the reaction zone.

\subsection{The Outflow from the Gaseous Reaction Zone}

Nelson et al[2] calculated the gaseous reaction zone volume using the ideal gas law. Furthermore extraction mechanisms in the reaction zone were ignored. When the sample ignited the reaction zone volume increased rapidly, extinguishing the flame. Consequently their model only applied to the pre-ignition and immediate post-ignition time 
periods. We follow Rychly and Costa[1] and assume that the gaseous reaction zone has fixed volume. In order to prevent the premature extinction of the flame extraction mechanisms are modelled.

The first extraction mechanism is a consequence of the solid-phase chemistry. There is a volumetric flow rate into the gaseous reaction zone associated with the degradation of the solid ( $\left.q_{\text {inflow }}\right)$, accordingly there is an outflow rate. The second extraction mechanism is a consequence of the gas-phase chemistry. The gas-phase reaction produces more moles of products than it consumes of reactants. Consequently it 'generates' volume, 'pushing out' material from the reaction zone; so that there is a volumetric outflow rate ( $\left.q_{\text {reaction }}\right)$ associated with the gaseous chemistry.

\section{MODEL EQUATIONS}

Although we use equation (3) to define the kinetics of the solid-phase degradation the model equations are defined using the traditional definition, equation (2), to increase the ease of understanding of our model.

$$
\begin{aligned}
\frac{\mathrm{d} \mathcal{M}_{1}}{\mathrm{dt}}= & -A_{s} \mathcal{M}_{1} \exp \left[\frac{-E_{s}}{R T_{s}}\right] \\
c_{p_{s}} \rho_{s} V_{s} \frac{\mathrm{d} T_{s}}{\mathrm{dt}}= & Q_{s} A_{s} \mathcal{M}_{1} \exp \left[\frac{-E_{s}}{R T_{s}}\right]+\alpha_{s}\left(1-\alpha_{g}\right) S \mathcal{L}-S \chi_{0}\left(T_{s}-T_{g}\right) \\
& +\sigma\left(\left[1-\mathcal{F}_{g . s}\right] \alpha_{s} T_{a}^{4}-\epsilon_{s} T_{s}^{4}\right) S+\alpha_{s} \mathcal{F}_{g . s} \epsilon_{g} \sigma T_{g}^{4} S, \\
\frac{\mathrm{d} \mathcal{M}_{2}}{\mathrm{dt}}= & \frac{q_{\text {inflow }}}{v_{p}}-\frac{q_{\text {inflow }} \mathcal{M}_{2}}{V_{g}}-A_{g} M_{2} \exp \left[\frac{-E_{g}}{R T_{g}}\right]-\frac{q_{\text {reaction }} \mathcal{M}_{2}}{V_{g}} \\
\frac{\mathrm{d} \mathcal{M}_{3}}{\mathrm{dt}}= & A_{g} M_{2} \exp \left[\frac{-E_{g}}{R T_{g}}\right]-q_{\text {inflow }} \frac{M_{3}}{V_{g}}-\frac{q_{\text {reaction }} \mathcal{M}_{3}}{V_{g}} \\
c_{p_{g}} \rho_{g} V_{g} \frac{\mathrm{d} T_{g}}{\mathrm{dt}}= & -Q_{s} A_{s} \mathcal{M}_{1} \exp \left[\frac{-E_{s}}{R T_{s}}\right]+Q_{g} A_{g} \mathcal{M}_{2} \exp \left[\frac{-E_{g}}{R T_{g}}\right] \\
& -S_{\chi_{1}}\left(T_{g}-T_{a}\right)-S \chi_{0}\left(T_{g}-T_{s}\right)-2 \delta_{1}(x+y) \chi_{2}\left(T_{g}-T_{a}\right) \\
& +2 \sigma \epsilon_{g} \delta_{1}(x+y)\left(T_{a}^{4}-T_{g}^{4}\right)+\sigma S\left(\alpha_{g} T_{a}^{4}-\epsilon_{g} T_{g}^{4}\right) \\
& +\sigma S\left(\left[1-\mathcal{F}_{s . g}\right] \alpha_{g} T_{a}^{4}-\epsilon_{g} T_{g}^{4}\right)+\alpha_{g} \epsilon_{s} \sigma \mathcal{F}_{s . g} T_{s}^{4} S+\alpha_{g} S \mathcal{L}(t) \\
& -c_{p_{g}} \rho_{g} T_{g} q_{\text {inflow }}-\frac{q_{\text {reaction }} T_{g}}{V_{g}}, \\
R_{p} & A_{s} \mathcal{M}_{1} \exp \left[-E_{s} / R T_{s}\right], \\
& \frac{R T_{s}}{\mathcal{W}_{2} \mathcal{P}^{0}}, \\
v_{p} & R_{p} \cdot v_{p}, \\
q_{\text {inflow }}, & {\left[\left(\frac{\mathcal{W}_{2}-\mathcal{W}_{3}}{\mathcal{W}_{2} \mathcal{W}_{3}}\right) \cdot \frac{R A_{g}}{\left.\mathcal{P}^{0}\right]} \exp \left[\frac{-E_{g}}{R T_{g}}\right] \mathcal{M}_{2} T_{g} .\right.}
\end{aligned}
$$

For simplicity we ignore consumption of the solid fuel, $\mathcal{M}_{1}$ is fixed to its initial value and equation (6) discarded. This assumption has negligible effect on the calculation of the critical heat flux for ignition[2] because the characteristic time for heat transfer is considerable shorter than that for mass changes. 
In analysing this model it is convenient to non-dimensionalise the variables. To that end we introduce non-dimensionalised temperatures $\left(T_{i}^{*}\right)$, non-dimensionalised 'concentrations' $\left(\mathcal{M}_{j}^{*}, \mathrm{j}=1,2,3\right)$ and a non-dimensionalised timescale $\left(t^{*}\right)$. For sake of brevity the non-dimensionalised equivalent of equations (6-10) are not provided, the definitions of the reduced variables are provided in the Nomenclature.

\section{RESULTS}

Equations (7-10) were integrated using a variable order, variable step, method, using the Backward Differentiation Formulae, written to solve stiff problems (routine D02EPF in the NAG Fortran Library). For path following the Auto 94 software was used[7].

\subsection{Calculating the Critical Heat Flux}

Nelson et al [2] found two mechanisms by which thermally thin thermoplastics autoignite. These were monotonic ignition, ignition occurs with a monotonic increasing gaseous temperature, and oscillatory ignition, in which ignition occurs with an oscillatory gaseous temperature. In this paper we investigate the phenomenon of monotonic ignition.

Calculation By Integration. The monotonic route to ignition is illustrated in Figure 2. When the irradiance is $18.4 \mathrm{kWm}^{-2}$ the system is subcritical and the nondimensionalised gas-phase temperature approaches a low valued steady-state. When the irradiance is increased to $18.5 \mathrm{kWm}^{-2}$ the system becomes supercritical and the non-dimensionalised gas-phase temperature approaches a much higher valued steadystate, corresponding to the existence of a steady flame over the sample. Note that the trajectories track each other with negligible difference until just prior to ignition. The critical heat flux for ignition is bounded between these values for the irradiance; from an experimental viewpoint the value of $\mathcal{L}_{c r}=18.5 \mathrm{kWm}^{-2}$ is sufficiently accurate.

Calculation By Path Following Methods. Auto 94 was used to trace the steady-state solution branches as a function of the non-dimensionalised irradiance. Figure 3 shows a typical result. The curve of stationary-states contains two limit points, where the path of steady-states turns around on itself, and two Hopf bifurcation points, where periodic orbits appear. The periodic orbits generated by the Hopf bifurcations in Figure 3 are unsteady and so are not observable experimentally, accordingly they are not shown. Although it is not experimentally meaningful to have a negative non-dimensionalised irradiance, those parts of the curve corresponding to such values are included to show that the steady-states form a continuous curve - the solution curve is not disjoint.

In Figure 3 it is the limit point in the positive quadrant that defines ignition. For low, positive, values of the non-dimensionalised irradiance (NDI) there is a low stable steady-state branch (as discovered by integration). At the limit-point there is a change in stability of the steady-states (the solid line changes to a dashed line). For values of the NDI greater than that corresponding to the limit point the only stable steady-state is on the ignition branch, so that the system ignites. The limit point corresponds to an irradiance of $18.431\left(\mathrm{kWm}^{-2}\right)$, which is in the region determined by integration.

When a bifurcation point of interest has been identified its location can be followed as a second parameter is varied. For instance, how does the critical heat flux depend upon the degradation kinetics? To investigate this the characteristic temperature is 


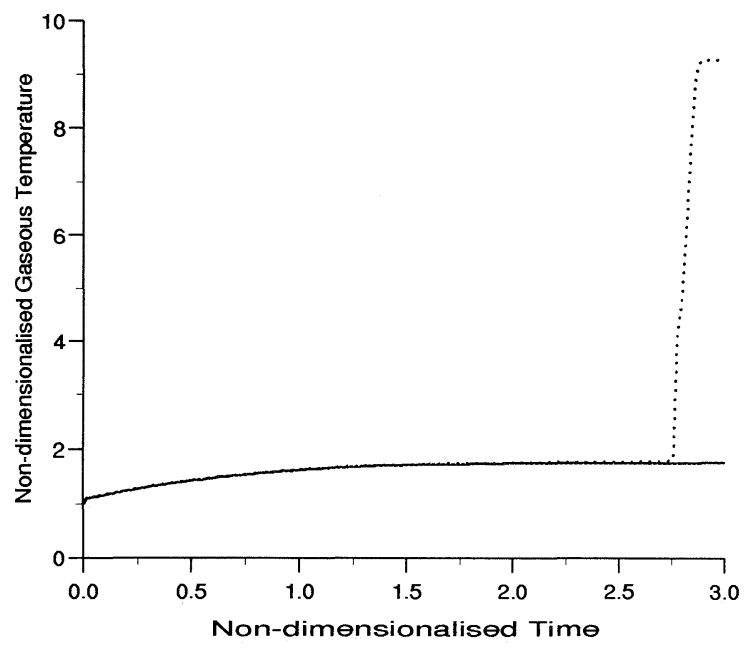

Figure 2: Monotonic ignition in the cone calorimeter. Variation of non-dimensionalised gaseous temperature with non-dimensionalised time. (a) $\mathcal{L}=18.4 \mathrm{kWm}^{-2}$ (solid line, subcritical), (b) $\mathcal{L}=18.5 \mathrm{kWm}^{-2}$ (dotted line, supercritical). Parameter values: characteristic temperature, $T_{c}=620(\mathrm{~K})$; solid phase activation energy, $E_{s}=80\left(\mathrm{kJmol}^{-1}\right)$. Note that a non-dimensionalised time of 3.0 corresponds to 200 seconds.

fixed and the solid-phase activation energy used as a secondary bifurcation parameter. The results are shown in Figure 4.

From Figure 4 the variation in the critical heat flux with activation energy is approximately $1.6 \mathrm{kWm}^{-2}$. This is smaller than the typical experimental error in determining this parameter, so that, for the range of activation energies considered, it is reasonable to define an averaged critical heat flux for ignition $\left(\overline{\mathcal{L}_{c r}}\right)$. This is calculated by integrating the curve shown in Figure 4, or formally by

$\overline{\mathcal{L}_{c r}}=\frac{10^{-3}}{160} \int_{E_{s}=80 \times 10^{3}}^{E_{s}=240 \times 10^{3}} \mathcal{L}_{c r}\left(E_{s}\right) \mathrm{d} E_{s}$.

When the characteristic temperature is $620 \mathrm{~K}$ we calculate that $\overline{\mathcal{L}_{c r}}=17.400\left(\mathrm{kWm}^{-2}\right) \pm$ $2.3 \%$. The variation in $\overline{\mathcal{L}_{c r}}$ with characteristic temperature is illustrated in Figure 5. The standard deviation decreases with increasing characteristic temperature.

\subsection{The Concept of A Critical Surface Temperature}

Thermal pyrolysis models have been used for many years and it is of considerable interest to see if this approximation can be obtained from a dynamical systems model.

Figure 6 shows five trajectories, one subcritical and four supercritical, obtained by integrating equations (7-10). Figure 3 shows the variation of non-dimensionised gaseous steady-state temperature with non-dimensionalised irradiance. A similar figure can be drawn for the non-dimensionalised solid-phase temperature, but for brevity is not included. Recall that the limit point in the positive quadrant defines ignition. Figure 3 


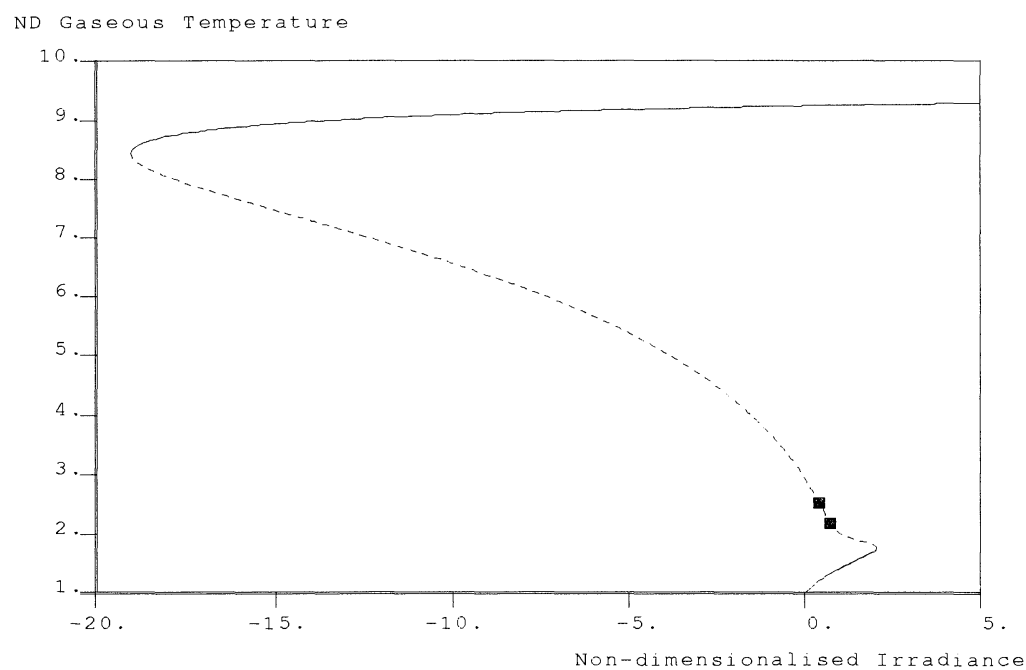

Figure 3: Variation of the non-dimensionalised steady-state gaseous temperature with non-dimensionalised irradiance. Solid Lines: stable stationary states; Dotted Lines: unstable stationary States; Squares: Hopf Bifurcation Points. parameter values: characteristic temperature $T_{c}=620(\mathrm{~K})$, solid phase activation energy $E_{s}=80\left(\mathrm{kJmol}^{-1}\right)$.

can also be interpreted as defining a maximum 'safe' steady-state gaseous temperature. Similarly there will be a maximum 'safe' solid-phase steady-state temperature; the steady-state temperature can not above this without ignition occurring.

Now consider Figure 6. Trajectory (1) corresponds to a subcritical irradiance, just below the critical value determined by path-following, so that the system evolves onto the lower steady-state branch. Trajectory (2) corresponds to a marginally supercritical irradiance. These trajectories are of negligible difference until the second ignites. Ignition occurs when the non-dimensionalised temperature reaches a value that is just higher than the steady-state temperature reached in the first trajectory.

As the heat-flux is increased further from criticality (trajectories 3-5) the trajectories increasingly diverge from the subcritical trajectory. However, in all cases, ignition occurs at a temperature that is only slightly higher than the steady-state value reached in trajectory (1). Hence not only is the concept of a thermal-pyrolysis temperature, at which ignition occurs, validated, the dynamical systems model also defines this temperature: it is the steady-state value at the limit point defining ignition. Accordingly, in a dynamical systems model the effect of additives upon the critical surface temperature can be calculated, something that can only be guessed at in more empirical approaches.

\section{DISCUSSION}

There are several ways in which the model presented in this paper can be expanded. For example, oxygen consumption in the reaction-zone is assumed to be negligible. A 


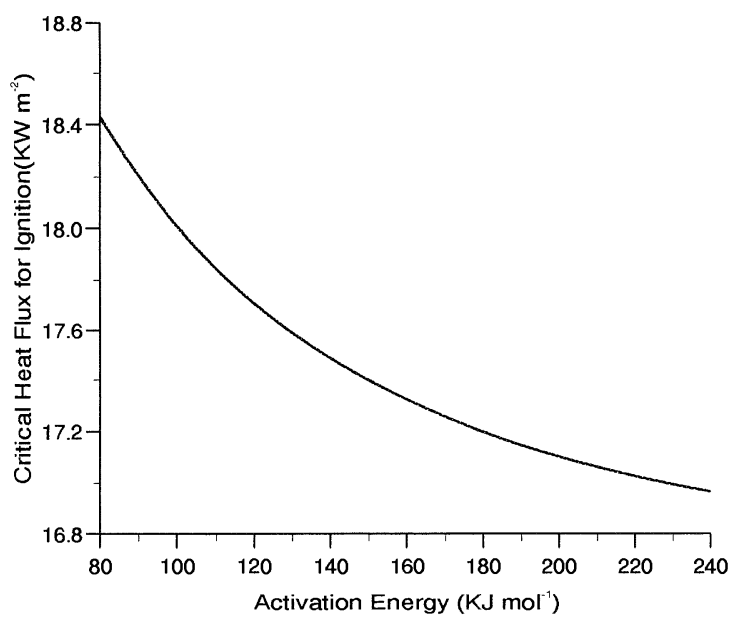

Figure 4: The variation of the critical heat flux for ignition with solid-phase activation energy (secondary bifurcation parameter) for fixed characteristic temperature. Parameter value: characteristic temperature $T_{c}=620(\mathrm{~K})$.

more important extension is to consider the ignition of non thermally thin materials: Equations (7) \& (12) being replaced by a reaction-diffusion equation and an integral over the sample thickness respectively.

The critical heat flux for ignition has been found by direct integration and by pathfollowing methods. The latter is considerable easier than the former and also locates other bifurcations. Although in this case the other bifurcations are not important this is not necessarily so. Hence path-following methods are not only quicker, they provide greater insight into the dynamics of the model.

Figure 3 shows the non-dimensionalised steady-state gaseous temperature as a function of the non-dimensionalised irradiance. Similar data is obtained for the other nondimensionalised variables. From these the steady-state peak rate of heat release can be calculated. This is higher than the transient value because fuel-consumption has been ignored. However, this provides qualitative information on peak heat release rates.

For fixed characteristic temperature we have shown that the critical heat flux for ignition does not vary greatly with activation energy and have defined an averaged critical value. The dependence of this quantity upon characteristic temperature has been investigated (Figure 5). This approach can be extended to include the dependence upon density and hence the functional form of the relationship between averaged critical heat flux and thermal inertia can be calculated. This would allow a comparison between a dynamical systems theory and simple thermal pyrolysis models.

In this paper we have investigated the dependence of critical heat flux upon degradation kinetics. The effect of other parameters of practical interest (fuel density, heat of gasification and the heat of combustion) can be calculated using path-following meth- 


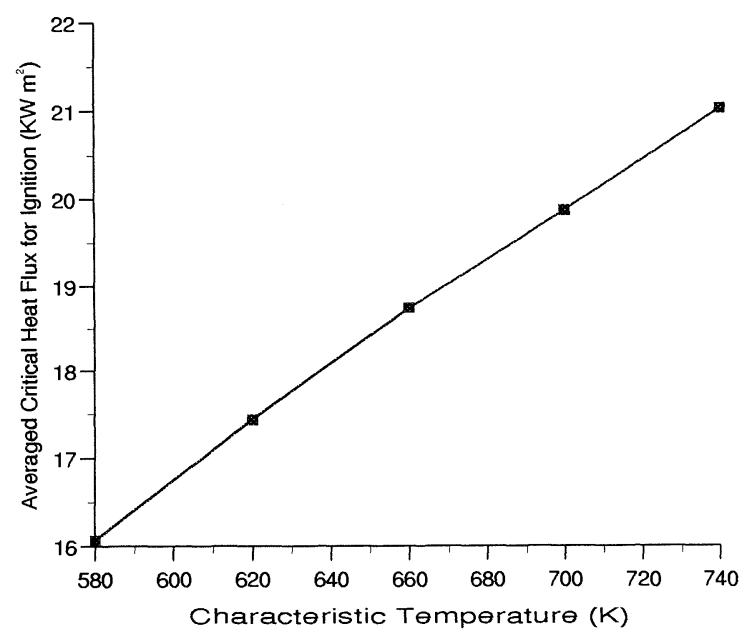

Figure 5: The variation of average critical heat flux for ignition $\left(\overline{\mathcal{L}_{c r}}\right)$ with the characteristic temperature $\left(T_{c}\right)$ of the sample.

ods. The gas-phase chemistry of certain additives can be modelled implicitedly, by using the heat of combustion as the control parameter, or explicitedly, by adding additional chemical reactions to the gas-phase model. Such an investigative approach into gasphase additives is not possible with other models of polymer ignition and is one of the attractions of a dynamical systems approach.

\section{CONCLUSIONS}

- The model of Nelson et al[2] has been revised to take into account extraction mechanisms operating in the gaseous reaction zone.

- The incorporation of these mechanisms has eliminated the problem of selfextinction of the flame.

- The phenomenon of monotonic ignition has been investigated by a combination of direct integration and path following methods.

- Monotonic ignition corresponds to a limit-point bifurcation.

- For fixed characteristic temperature, the critical heat flux does not depend greatly upon solid-phase activation energy.

- An upper bound on the peak heat release rates can be calculated from the bifurcation diagram.

- The concept of a critical surface temperature defining criticality has been validated within the dynamical systems model. This critical value is given by the steadystate temperature at the limit-point governing ignition. 


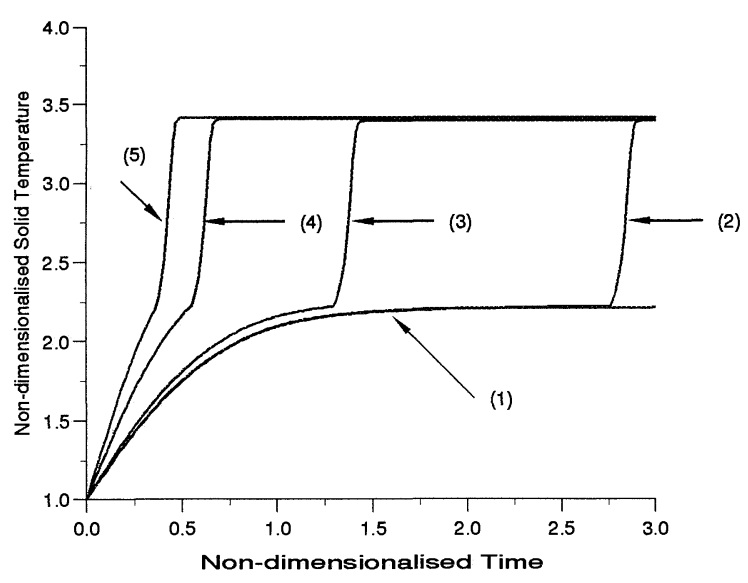

Figure 6: The variation of non-dimensionalised solid temperature with nondimensionalised time. The non-dimensionalised irradiances correspond to heat-fluxes of: (1) $18.4 \mathrm{kWm}^{-2}$ (subcritical), (2) $18.5 \mathrm{kWm}^{-2}$, (3) $20.0 \mathrm{kWm}^{-2}$, (4) $30.0 \mathrm{kWm}^{-2}$, (5) $40.0 \mathrm{kWm}^{-2}$. Parameter values: characteristic temperature $T_{c}=620 \mathrm{~K}$; solid phase activation energy, $E_{s}=80 \mathrm{kJmol}^{-1}$.

\section{REFERENCES}

1. Rychly, J., and Costa, L., "Modelling of Polymer Ignition and Burning Adopted for Cone Calorimeter Measurements: The Correlation between the Rate of Heat Release and Oxygen Index", Fire and Materials, 19, 215-220, 1995.

2. Nelson, M.I., Brindley, J., and McIntosh, A.C., "A Mathematical Model of Ignition in the Cone Calorimeter", Combustion Science and Technology, 104, 35-54, 1995.

3. Nelson, M.I., Brindley, J., and McIntosh, A.C., "The Dependence of Critical Heat Flux on Fuel and Additive Properties: A Critical Mass Flux Model", Fire Safety Journal, 24:2, 107-130, 1995.

4. Murray, P., and White, J., "Kinetics of the Thermal Dehydration of Clays. Part IV. Interpretation of the Differential Thermal Analysis of the Clay Minerals", Transactions of the British Ceramic Society, 54, 204-238, 1955.

5. Seydel, R., "Tutorial On Continuation", International Journal of Bifurcation and Chaos, 1:1, 3-11, 1991.

6. Seydel, R., Practical Bifurcation and Stability Analysis: From Equilibrium to Chaos, 1st ed, Springer-Verlag, New York, 1994.

7. Doedel, E., Wang, X., and Fairgrieve, T., "AUTO 94 Software for Continuation and Bifurcation Problems in Ordinary Differential Equations", Applied Mathematics Report California Institute of Technology Pasadena, California 91125, November 1994. 